\title{
PMIP4 contribution to CMIP6
}

\author{
Qiong Zhang ${ }^{1}$, J.C. Hargreaves ${ }^{2}$, P. Braconnot ${ }^{3}$ and M. Kageyama ${ }^{3}$ \\ 1st PMIP4 conference, Stockholm, Sweden, 25-29 September 2017
}

Paleoclimate Modelling

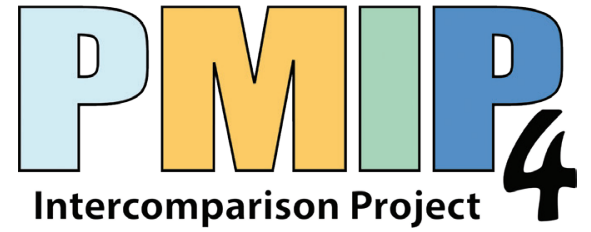

The scientific focus of the Paleoclimate Modelling Intercomparison Project (PMIP) is to understand the response of the climate system to different forcings that have occurred at various periods prior to the historical instrumental record. Climate models are primarily constructed to understand modern climate and predict future climate evolution. These models are developed based on scientific understanding gained from studying the climate of the recent past. Past periods when the climate was radically different provide a useful test of model performance. The quantity of observational information on past climate, based on physical, chemical and biological records, is also increasing, providing more opportunities for quantitative evaluation of models.

PMIP is now in its fourth phase. Five of the PMIP4 experiments are included within the Coupled Model Intercomparison Project Phase 6 (CMIP6), and a number of the PMIP4 working groups are organizing additional experiments covering a broader range of topics (Fig. 1). The results from all of the experiments will provide a substantial contribution to the Intergovernmental Panel on Climate Change Sixth Assessment Report (IPCC AR6). New analyses will focus on climate sensitivity, changes in the hydrological cycle, physical and biogeochemical feedbacks, climate variability, and the credibility of the climate models which are used for future climate projections.

This first PMIP4 conference brought together 160 researchers from 24 countries. New, indepth analyses from PMIP3 were presented as well as some first results from the new PMIP4 simulations. All participants gave a short oral presentation so that attendees gained an overview of all the ongoing activities, and to focus discussions at the subsequent poster sessions. A number of new initiatives in data synthesis are underway, and these were given slightly longer oral presentations. Eight speakers from outside the PMIP community gave stimulating, perspective talks. The last day of the conference was devoted to the connection of PMIP4 to CMIP6 and IPCC AR6. For the IPCC AR6, paleoclimate science will be included throughout the report rather than being an isolated chapter. Discussions considered how the key foci of PMIP4 could contribute to the different AR6 chapters where paleoclimate is relevant.

The conference provided opportunities for early-career scientists to develop networks within PMIP. Several of them led activities and discussions, and proposed to (1) have more synergy and interaction between PMIP and PAGES; (2) bring water isotope modeling into PMIP experiments, and make it a standard output; (3) use proxy system models (e.g. PRYSM) for all model-proxy comparisons using PMIP data; and (4) develop a standard approach to synthesize the model and proxy data in the PMIP community, to ensure scientists outside of each respective community can use the data in an appropriate way.

\section{ACKNOWLEDGEMENTS}

The PMIP4 conference was co-sponsored by PAGES, Swedish Research Council and the Bolin Centre for Climate Research at Stockholm University.

\section{AFFILIATIONS}

Department of Physical Geography and Bolin Centre for Climate Research, Stockholm University, Sweden ${ }^{2}$ BlueSkiesResearch, Settle, UK

${ }^{3}$ LSCE/IPSL, CEA-CNRS-UVSQ, Université Paris-Saclay, France

\section{CONTACT}

Qiong Zhang: qiong.zhang@natgeo.su.se REFERENCES

Haywood A et al. (2016) Clim Past 12: 663-675 Jungclaus J et al. (2017) Geosci Model Dev 10: 4005-4033 Kageyama M et al. (2016) Geosci Model Dev Discuss Kageyama M et al. (2017) Geosci Model Dev 10: 4035-4055

Otto-Bliesner B et al. (2017) Geosci Model Dev 10: $3979-4003$

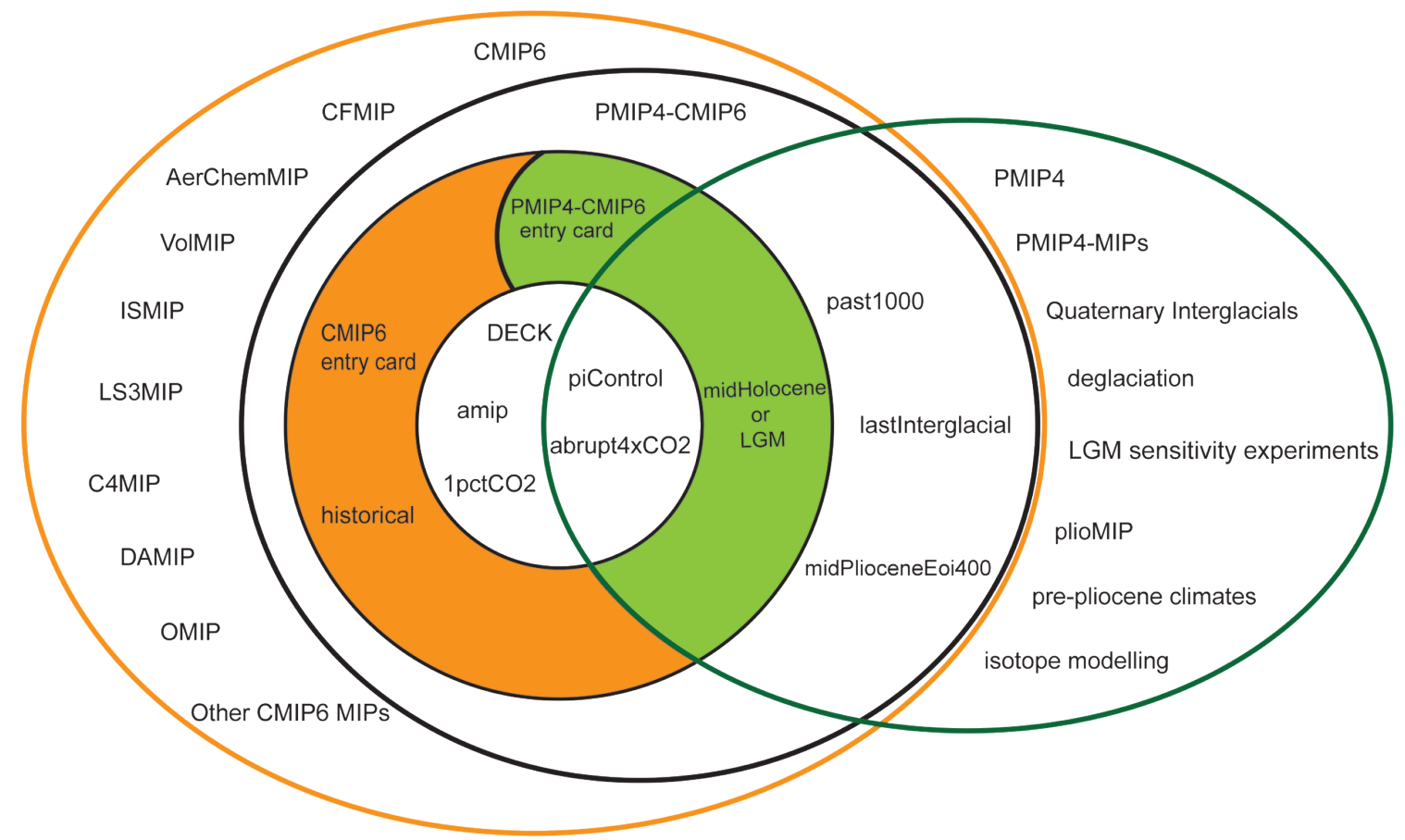

Figure 1: The PMIP4/CMIP6 experiments in the framework of CMIP6, link with other MIPs, and in the framework of PMIP4, with its working groups (Kageyama et al. 2016). The overview and overarching analyses plan for PMIP4 experiments are documented in Kageyama et al. (2016), the series of PMIP4 protocol documents on scientific objectives and experiment design include the two interglacials (Otto-Bliesner et al. 2017), the Last Millennium (Jungclaus et al. 2017), the Last Glacial Maximum (Kageyama et al. 2017), and mid-Pliocene (Haywood et al. 2016). 